\title{
The structure of the ISM in the Zone of Avoidance by high-resolution multi-wavelength observations
}

\author{
L. V. Tóth ${ }^{1,2}$ Y. Doi ${ }^{3}$, S. Pinter ${ }^{1}$, T. Kovács ${ }^{1}$, S. Zahorecz ${ }^{4,5}$, \\ Z. Bagoly ${ }^{1}$, L. G. Balázs ${ }^{1,2}$, I. Horvath ${ }^{6}$, I. I. Racz ${ }^{1,2}$ and T. Onishi ${ }^{4}$ \\ ${ }^{1}$ Eötvös University, Budapest, \\ email: 1.v.toth@astro.elte.hu \\ ${ }^{2}$ MTA CSFK Konkoly Observatory, Budapest \\ ${ }^{3}$ Department of Earth Science and Astronomy, The University of Tokyo, 3-8-1 Komaba, \\ Meguro-ku,Tokyo 153-8902, Japan \\ ${ }^{4}$ Osaka Prefecture University, 1-1 Gakuen-cho, Naka-ku, Sakai, Osaka 599-8531, Japan \\ ${ }^{5}$ Chile Observatory, NAOJ, NINS, 2-21-1 Osawa, Mitaka, Tokyo 181-8588, Japan \\ ${ }^{6}$ National University of Public Service, Budapest
}

\begin{abstract}
We estimate the column density of the Galactic foreground interstellar medium (GFISM) in the direction of extragalactic sources. All-sky AKARI FIS infrared sky survey data might be used to trace the GFISM with a resolution of 2 arcminutes. The AKARI based GFISM hydrogen column density estimates are compared with similar quantities based on HI $21 \mathrm{~cm}$ measurements of various resolution and of Planck results. High spatial resolution observations of the GFISM may be important recalculating the physical parameters of gamma-ray burst (GRB) host galaxies using the updated foreground parameters.
\end{abstract}

Keywords. ISM: structure, infrared: ISM, gamma-ray burst: general, GRB110213A

\section{Introduction}

Gamma-ray bursts (GRBs) are the most energetic events in the Universe. Longduration GRBs (LGRB) are associated with the explosions of massive stars (e.g. Stanek et al. 2003) and they are expected to trace ongoing star-formation in galaxies. The number of GRBs with known spectroscopic redshift is still too low to link huge GRB structures such as the Giant Ring of GRBs (with a mean angular diameter of $36 \mathrm{deg}$ corresponding to $1720 \mathrm{Mpc}$ in the comoving frame, Balázs et al. (2015)) to patterns in the galaxy distribution. GRBs are seen up to a redshift of 9.4 (based on photometric redshift, GRB090429B; Cucchiara et al. (2011). Thus we expect some insight even to the re-ionization era as well, using LGRBs as tools to probe star-formation activity in the distant universe. The intrinsic hydrogen column density $N(\mathrm{H})_{\text {GRB }}$ may be derived from the extinction seen in the X-ray and optical (see eg. Fynbo et al. (2009)) afterglows. An estimate on the $N(\mathrm{H})_{\mathrm{MW}}$ galactic foreground hydrogen column density towards the GRB is however part of the calculations. Foreground estimations are typically based on galaxy counts (Burstein \& Heiles (1982)), HI 21cm surveys (mostly the LAB survey Kalberla et al. (2005)), extinction or reddening maps calculated from infrared surveys (eg. Schlafly \& Finkbeiner (2011)), or from individual spectroscopic measurements and colors of nearby Galactic stars. 

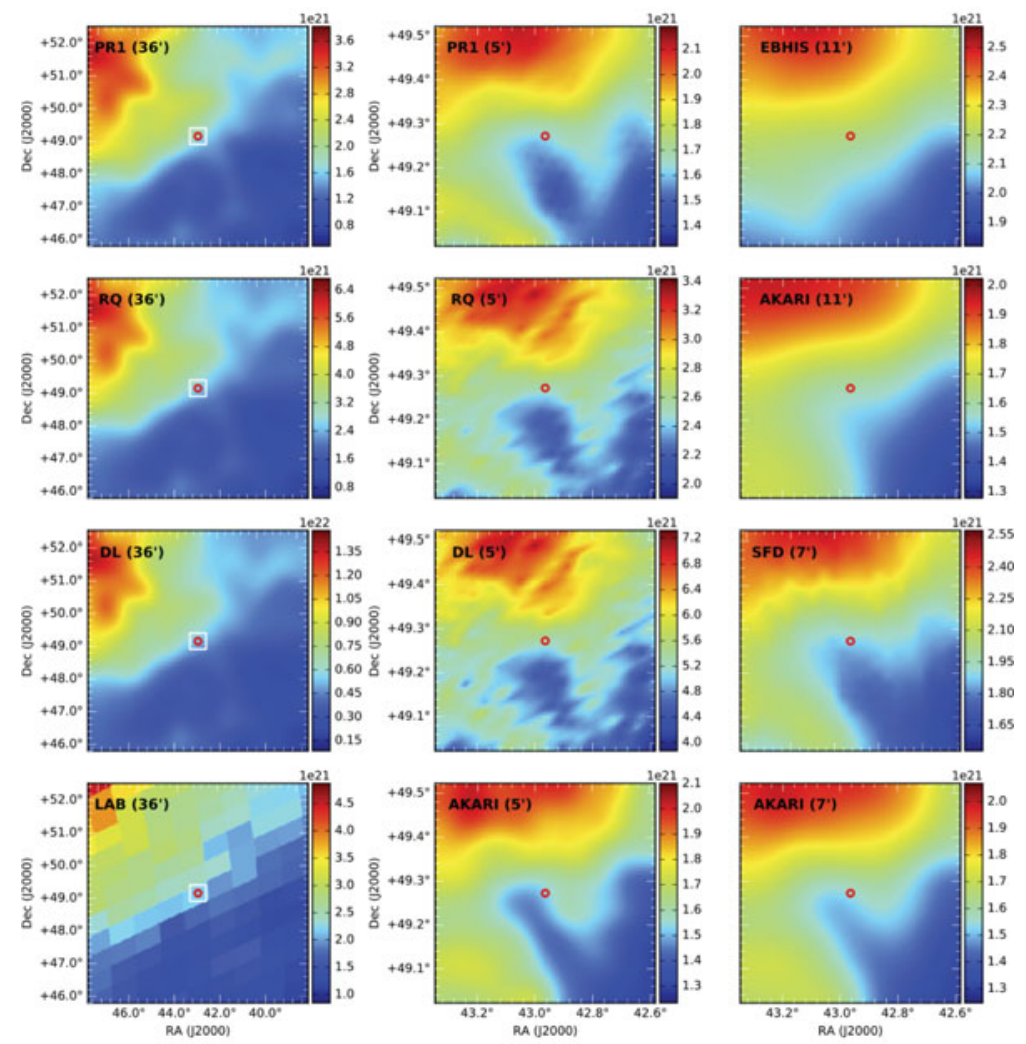

Figure 1. The $N(\mathrm{H})_{\mathrm{MW}}$ galactic foreground hydrogen column density distribution in the direction of GRB 110213A, based on various all sky surveys. Left column: The first three images from top are Planck PR1, Planck PR2 RQ, and Planck PR2 DL based $N(\mathrm{H})_{\mathrm{MW}}$ distributions smoothed to 36 ' resolution; while at the bottom is the $N(\mathrm{H})_{\mathrm{MW}}$ from the LAB survey. Middle column: All images show the central area of the left column (marked with white box), but with a resolution of $5^{\prime}$. The first three images from top are similar to the left column, the bottom one is based on AKARI FIS data. Right column: The first two from the top are $N(\mathrm{H})_{\mathrm{M} W}$ with $11^{\prime}$ resolution based on EBHIS and AKARI. The lower two are based on the SFD and AKARI with $7^{\prime}$ resolution both. See also text.

\section{Analysis and results}

\subsection{Analysis of the AKARI FIS All Sky Survey images}

$A K A R I$ FIS full sky FIR images were recently published by Doi et al. (2015) with a detection limit of about $<10 \mathrm{MJysr}^{-1}$ and a relative photometric accuracy of about $<20 \%$. We substracted 30 by $30 \square^{\prime}$ images centered on GRBs with known spectroscopic redshifts from Horváth et al. (2014), and smoothed those to $2^{\prime}$ spatial resolution to calculate hydrogen column densities as described in Toth et al.(2017).

\subsection{Other all-sky data}

For comparison we used column density maps derived from several lower resolution all-sky data, like IR dust emission or extinction maps such as from Schlafly \& Finkbeiner (2011) and from Planck results Planck Collaboration et al. (2014) and Planck Collaboration et al. (2016), as well as from neutral hydrogen measurements like the LAB Kalberla et al. (2005), and EBHIS Kerp et al. (2011) surveys. 

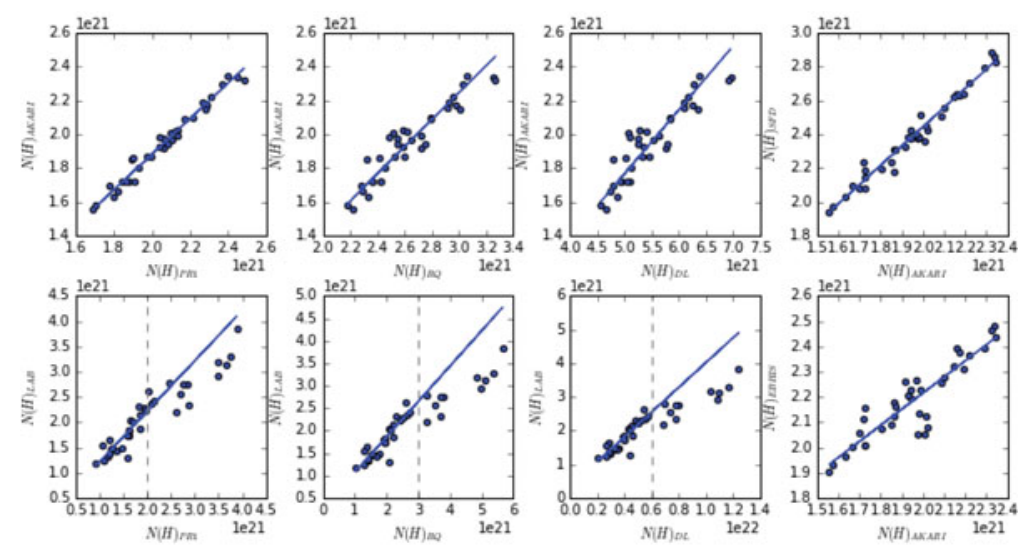

Figure 2. Correlation plots of the estimated $N(\mathrm{H})_{M W}$ galactic foreground hydrogen column densities in the direction of GRB110213A, based on various all sky surveys. Top row from left to right: AKARI vs. Planck PR1; AKARI vs. Planck PR2 RQ; AKARI vs. Planck PR2 DL; SFD vs. AKARI. The bottom row: LAB survey vs. Planck PR1; LAB survey vs. Planck PR2 RQ; LAB survey vs. Planck PR2 DL; EBHIS vs. AKARI. Ordinary least square fitted lines are drawn. The Pearson's correlation coefficients and the least square fits were calculated only at the linear parts of the correlation plots with HI data, the limits are indicated (dashed lines).

\subsection{GRB110213A and its Galactic foreground}

GRB110213A (D'Elia et al. (2011)) is an LGRB ( $T_{90}=48 \mathrm{~s}$, Cucchiara et al. (2011)), its Swift X-ray afterglow light curve contains four breaks. The X-ray afterglow spectrum is a nice power-law continuum modulated by absorption. The $N(\mathrm{H})_{\mathrm{MW}}$ galactic foreground hydrogen column density distribution in the direction of GRB110213A is shown in Fig.1 based on various all sky surveys.

Correlation plots of the estimated $N(\mathrm{H})_{M W}$ galactic foreground hydrogen column densities in the direction of GRB110213A, based on various all sky surveys is shown in Fig.2 based on various all sky surveys.

The AKARI based high resolution $N(H)_{\mathrm{MW}}$ is seen in Fig.3. We note the difference between the UKSSDC estimate of $N(\mathrm{H})_{\mathrm{MW}}=3.4 \times 10^{21}$ (based on LAB), and ours based on the AKARI data of $N(\mathrm{H})_{\mathrm{MW}}=2.4 \times 10^{21}$

\subsection{Intrinsic hydrogen column density at GRB110213A}

An X-ray afterglow spectrum of GRB110213A from the Swift-XRT GRB Catalogue†, was analyzed with the Xspec $\ddagger$ (Arnaud (1996)). The same model was used as in the automatic analysis by UKSSDC (Evans et al. (2009)). The spectrum (see Fig. 3) was fitted with an absorbed power law with two absorbing components: the Galactic foreground, and the intrinsic one. While the first one was held fixed during the fit, the excess hydrogen column of the second component was resulted by the fit. The UKSSDC estimate of $N(\mathrm{H})_{\mathrm{GRB}}=3.6 \times 10^{20}$ was unlikely low, but based on the high resolution AKARI data we get a more realistic value of $N(\mathrm{H})_{\mathrm{GRB}}=7.4 \times 10^{21}$.

\section{Conclusion}

Compared to the over 100 cases we analyzed, the case of GRB110213A shows an almost typical pattern of the Galactic foreground, i.e. it is shallower in the given direction than it

$\dagger$ maintained by the UK Swift Science Data Centre (UKSSDC)

$\ddagger$ Xspec is part of the HEASOFT Software package of NASA's HEASARC 

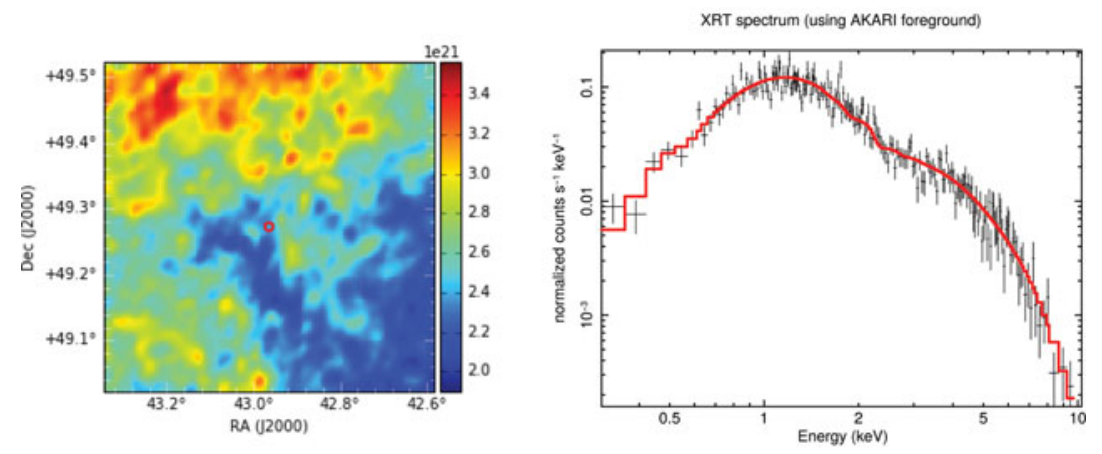

Figure 3. Left: AKARI based $N(\mathrm{H})_{\mathrm{MW}}$ map (calibrated with RQ). Right: XRT spectrum of GRB110213A fitted using the AKARI based Galactic foreground.

would be estimated from low resolution surveys. The use of our high resolution $N(H)_{M W}$ in the XRT spectral fits resulted in a $2060 \%$ relative difference in the $N(\mathrm{H})_{\text {GRB }}$ values compared to the UKSSDC estimate.

\section{Acknowledgements}

This work was supported by the OTKA NN-111016 grant; the NAOJ ALMA Scientific Research Grant Number 2016-03B; and the Hungarian New National Excellence Program of the Ministry of Human Capacities (TK and IR). Based on observations with AKARI, a JAXA project with the participation of ESA, and observations obtained with Planck (http://www.esa.int/Planck), an ESA science mission with instruments and contributions directly funded by ESA Member States, NASA, and Canada. This research has made use of the VizieR catalogue access tool, CDS, Strasbourg, France. This research has made use of the data supplied by the UK Swift Science Data Centre at the University of Leicester, and the SIMBAD database, operated at CDS, Strasbourg, France.

\section{References}

Arnaud, K. A. 1996, Astronomical Data Analysis Software and Systems V, 101, 17

Balázs, L.G. et al. 2015 MNRAS, 452, 2236

Burstein, D. \& Heiles, C. 1982, AJ, 87, 1165

D'Elia, V. et al. 2011, GCN circ, 11705

Cucchiara, A. et al. 2011 ApJ, 743, 154

Doi, Y., Takita, S., Ootsubo, T., et al. 2015, PASJ, 67, 50

Evans, P. A., Beardmore, A. P., Page, K. L., et al. 2009, MNRAS, 397, 1177

Fynbo, J.P.U. et al. 2009 ApJS, 743, 154

Horváth, I., Hakkila, J., \& Bagoly, Z. 2014, A\& A, 561, L12

Kalberla, P. M. W., Burton, W. B., Hartmann, D. et al. 2005, A\& A, 440, 775

Kerp, J., Winkel, B., Ben Bekhti, N., Flöer, L., \& Kalberla, P. M. W. 2011, AN, 332, 637

Planck Collaboration, Abergel, A., Ade, P. A. R., et al. 2014, A\&\&A, 571, A11

Planck Collaboration, Adam, R., Ade, P. A. R., et al. 2016, A\& A, 594, A10

Schlafly, E. F. \& Finkbeiner, D. P. 2011, APJ, 737, 103

Stanek, K. Z. et al. 2003 ApJ, 80976

Toth, L. V., Doi, Y., Zahorecz, S., et al. 2017, PKAS, 32, 113 